\title{
Equity-Oriented Train Timetabling with Collaborative Passenger Flow Control: A Spatial Rebalance of Service on an Oversaturated Urban Rail Transit Line
}

\author{
Chaoqi Gong $\mathbb{D}^{1},{ }^{1}$ Baohua Mao $\mathbb{D}^{1,2}{ }^{1,2}$ Min Wang, ${ }^{1}$ and Tong Zhang ${ }^{1}$ \\ ${ }^{1}$ Key Laboratory of Transport Industry of Big Data Application Technologies for Comprehensive Transport, \\ Beijing Jiaotong University, Beijing 100044, China \\ ${ }^{2}$ Integrated Transportation Research Centre of China, Beijing Jiaotong University, Beijing 100044, China
}

Correspondence should be addressed to Baohua Mao; bhmao@bjtu.edu.cn

Received 18 May 2020; Revised 13 July 2020; Accepted 10 September 2020; Published 23 September 2020

Academic Editor: Luigi Dell'Olio

Copyright (C) 2020 Chaoqi Gong et al. This is an open access article distributed under the Creative Commons Attribution License, which permits unrestricted use, distribution, and reproduction in any medium, provided the original work is properly cited.

On an oversaturated urban rail transit line, passengers at downstream stations have to wait for more trains until they get aboard, resulting in service imbalance problem. To improve the service quality, this paper proposes an integrated optimization approach combining the train timetabling and collaborative passenger flow control, with the aim of minimizing indicators associated with the passenger service imbalance and train loading capacity utilization. Considering train regulation constraints and passenger loading dynamics, a mixed-integer linear programming model is formulated. Based on the linear weighting technique, an iterative heuristic algorithm combining the tabu search and Gurobi solver is designed to solve the proposed model. Finally, a simple case with different-scale instances is used to verify that the proposed algorithm can obtain near-optimal solution efficiently. Moreover, a real-world case of Beijing Subway Batong Line is implemented to compare performances of the proposed approach with those under the original timetable and noncollaborative passenger flow control.

\section{Introduction}

In many large cities worldwide, close attention has been paid to public transportation in order to alleviate traffic congestion and reduce pollution. Especially, the urban rail transit system develops rapidly as its large capacity, high efficiency, reliability, safety, and low emission. Nevertheless, with passenger demands increasing unprecedentedly, some systems are suffering great pressure. For example, the passenger volume of metro networks both in Beijing and Shanghai has exceeded ten million per day [1].

Typically, the passenger arrival flow is extremely large during the morning and evening peak hours on workdays, in which overwhelming commuters are transported. Oversaturation occurs on an urban rail transit line when the train capacity is insufficient to satisfy the large travel demands over short periods of time; as a consequence, some passengers cannot board the first train and have to queue up on platforms to wait for the following available trains. From the welfare perspective of public transportation, operators are supposed to strive for guaranteeing an equitable provision of urban rail transit services, and equal individuals and groups should be treated the same in the distribution of transport resources (i.e., train capacity) $[2,3]$. However, in the aforementioned oversaturated situation, different passengers obtain different service qualities at the same cost (i.e., ticket fare), and sometimes, the gap can differ a lot. Specifically, the train will be soon overloaded when the passenger arrival volume at upstream stations is continuously large, causing passengers at downstream stations possibly waiting for several trains until they can get on board successfully. The distribution of train capacities is inequitable for passengers at downstream stations of a line, as they suffer more missed trains. Such service imbalance problem is ubiquitous during peak hours and may lead to complainants among passengers due to the long waiting time, which could belong to a subsidiary category of social inequity matters. 
Increasing the capacity is a straightforward solution to mitigate the service imbalance problem. But it is always infeasible because of long construction cycle, line layout conditions, and limited amount of available rolling stocks. Under such circumstances, one potential operation approach is optimizing train timetables, which often takes total passenger waiting time or average waiting time as the objective function to optimize train headways, routes, or stop patterns based on dynamic passenger demands. In reality, when the optimal timetables still cannot satisfy the passenger demands, passenger flow control is a supplementary measure. By lowering the speed and flow rate of passengers entering platforms at upstream stations, the capacity of each train departing from upstream stations can be reserved for passengers at subsequent stations, according to spatiotemporal passenger demands. Consequently, the passengers waiting at downstream stations can get aboard with missing fewer trains.

To our knowledge, the majority of current researches focus on the above two methods separately due to the complexity of the integrated problem. However, passenger flow control is closely related to the practical timetables, and in turn, the designed train schedules will greatly influence the implement of passenger flow control strategies. Additionally, handling the train timetabling and passenger flow control separately cannot cope with the service imbalance problem well on an oversaturated urban rail line. Hence, this paper intends to explore the integrated optimization of the train timetabling and passenger flow control to redistribute train capacities for each station and improve the overall equity performance on an oversaturated urban rail transit line.

The main contributions of this paper are as follows. First, the service imbalance problem caused by the supply-demand mismatch under oversaturated situation is analyzed. The equity performance is measured by an imbalance indicator associated with the number of missed trains. Second, to improve the system-wide service quality, a mixed-integer linear programming model with respect to the train timetabling and passenger flow control is formulated, aiming to minimize the imbalance indicator as well as balance the train loading capacity utilization. Third, based on the linear weighting method, an iterative heuristic algorithm integrating the tabu search and Gurobi solver is proposed to solve the two-objective model. And a simple case with different-scale instances and the real-world operation data of Beijing Subway Batong Line are implemented to demonstrate the efficiency and effectiveness of the proposed approach.

The remainder of the paper is organized as follows. Section 2 gives a review of relevant research work. Section 3 formulates the integrated optimization model of the train timetabling and passenger flow control in a mathematical way. Section 4 proposes a hybrid iterative heuristic algorithm to solve the integrated problem. Section 5 conducts a simple case and a real-world case of Beijing Subway Batong Line to demonstrate performances of the proposed approach. Section 6 draws the conclusions of this paper.

\section{Literature Review}

Equity primitively refers to social matters [4]. Increasingly, equity is becoming a long-term objective in urban transportation plans, and transport equity analysis has been conducted by many scholars [5]. The majority of these researches focus on revealing the potential connectivity provided by the current transport systems to different land uses and groups of residents through using accessibility measures from the macroscopic perspective [6-8]. Based on accessing the equity of the current transport systems, their studies intend to provide references for corresponding transport planners and policymakers. However, realizing the transport equity is related to not only the transport planning but also operations, in which transport services are embodied ultimately. For operations management, the focus of equity might be more targeted and narrowed down to a spatial balance of service on a rail line or bus route. In recent years, the equity of operations management has gradually received attention in rail and air services $[9,10]$, while studies focusing on operational equity performance of urban rail transit networks are insufficient.

As a key component of urban rail transit operations, train timetabling can improve the overall service quality and equity performance to some extent. Lots of scholars have provided excellent contributions on different optimization problems in the field of train timetabling. In general, train timetabling mainly aims to optimize the arrival and departure times of each train at each station. In the process of timetabling, various feasible methods, such as heterogeneous headways, short-turning routes, skip-stop pattern, and express/local mode, are considered to reduce the comprehensive cost of operators and passengers. Barrena et al. [11] studied the design and optimization of nonperiodic train timetabling adopted to a dynamic demand environment, with an aim of minimizing the average waiting time per passenger. Their results showed that nonperiodic timetables can yield improvements in the passenger average waiting time with respect to the regularly periodic cases. Furthermore, Barrena et al. [12] applied the nonperiodic timetable to a rapid rail line. They formulated two nonlinear mathematics and introduced a fast-adaptive large neighborhood search metaheuristic for large-scale instances. Sun et al. [13] built three formulations to design capacitated demandsensitive peak/off-peak timetables on the concept of equivalent time. Performances of these proposed models were evaluated on a metro line in Singapore. Niu and Zhou [14] focused on train timetabling on a heavily congested urban rail corridor and developed a nonlinear optimization model. In order to describe effective passenger loading time periods, the latest arrival time of boarded passengers was introduced. A local algorithm using cumulative input-output diagrams and the genetic algorithm were presented to solve individual cases and multistation problems, respectively. Gao et al. [15] proposed a train timetable rescheduling model in the case of a metro disruption. In this model, the skip-stop patterns were considered to speed up the circulation of trains and ease the platform pressure resulting from numerous stranded passengers faster. Shang et al. [16] 
derived an equity-oriented timetable model based on the multicommodity flow formulation, in which skip-stop patterns and FIFO principles were considered to portray the passenger boarding process. A linear programming model was formulated to reduce the number of passengers suffering the maximum number of missed trains and was decomposed into two subproblems by using Lagrangian relaxation framework. Li et al. [17] developed a mixed-integer nonlinear model, incorporating the express/local mode and train schedule, to minimize passenger travel time. In this model, two train services were provided, and overtaking was allowed at some specific locations, such as sliding and crossing tracks. With a joint aim of minimizing passenger waiting time and operational costs, as well as balancing train loads, Li et al. [18] established an MINP model combining short-turning strategies and heterogeneous headways for a congested urban rail line. Zhou et al. [19] proposed an approach for the energy-efficient timetable scheduling to minimize the net energy consumption considering utilizing regenerative energy. In addition, Gallo et al. [20] examined the problem of designing metro frequencies under the assumption of elastic passenger demands, in which the split of passenger demands among different transport modes depends on the corresponding service quality.

Commonly, in oversaturated circumstances (i.e., morning and evening peak hours), optimally designed timetables still cannot fully satisfy the temporally large passenger demands. Thus, passenger flow control is highly needed, and it has already been taken by some Chinese metro systems to balance the safety and efficiency for passengers. For example, ninety-six stations in Beijing metro network use inflow control strategies during the peak hours in their daily operations [21]. Unfortunately, the currently adopted control methods are usually implemented by the subjective judgements and experiences of operation staffs. The determination of control stations, inflow volume at each station, and time to carry out control strategies is lack of mathematical formulations [22]. Based on this, the optimization of passenger flow control attracts an ongoing interest of scholars. At the microscopic level, lots of studies focus on simulating passenger behaviors and evaluating the facility capacity of different layouts at a single station [23-25]. For instance, Xu et al. [24] extended the concepts of the station service capacity into three in different demand scenarios, in which the volume and ratio of inbound, outbound, and transfer passengers are uncertain. Mathematical models were put forward to evaluate the above three station capacities, and a uniform simulation-based algorithm was presented to find the optimal flow control volumes for different passenger types. Such station-based flow control methods neglect the potential collaboration among stations along the same line, or in a network, as a result, some studies tried to develop coordinated passenger flow control approaches. Wang et al. [26] built an integer programming model to achieve the optimal flow control state for a metro line, with an objective function of minimizing average passenger delay time. Jiang et al. [22] proposed a novel reinforcement learning-based method to optimize the coordinated inflow volume at each station. Additionally, Yang et al. [27] integrated passenger flow control and busbridging services as a compound strategy, and a two-stage MINP model was formulated. In this model, stations and time periods for implementing passenger controls were determined in the first stage, and then the second stage allocated the optimal bus-bridging services. At the network level, Zou et al. [28] established a traffic assignment model without capacity limits first to distribute the passenger flow on a metro network and then the target control stations; time and strength were determined through identifying capacity bottlenecks by considering the transport capacity of each interstation segment. Xu et al. [29] proposed a bilevel programming to regulate volumes of inbound and transfer passengers, in which the upper level was to optimize the control strategies, while the lower level was to redistribute passengers in metro networks. Shi et al. [30] proposed an integer linear programming model for the network passenger flow control problem to jointly minimize the total passenger waiting time and passenger accumulation risks at all stations.

We note that the above researches mainly focus on the train timetabling and passenger flow control separately. In recent years, a few scholars have attempted to investigate a joint optimization method for the dynamic train timetabling and passenger flow control with different objectives. Such objectives are usually relevant to passengers and operators, such as minimizing the total passenger waiting time [31], minimizing the number of stranded passengers [32], minimizing the amplitude of control action [33], or minimizing the timetable and headway deviations [34]. Here, it is worth pointing out that, although some researchers have studied the integrated problem of the train timetabling and passenger flow control from different perspectives, to our knowledge, the equity performance on an oversaturated urban rail line and equilibrium of train loading capacity are quite limited in the literature.

\section{Mathematical Formulation}

This paper considers an oversaturated urban rail transit line with heterogeneous headways and passenger flow control strategies, as shown in Figure 1. The stations are numbered as 1 to $m$ sequentially. Since there are two directions operating on different tracks and passenger demands and train operations in different directions are relatively independent to each other, this study only considers the trains and passengers in the more congested direction from station 1 to station $m$. Furthermore, for the purpose of implementing inflow control strategies, a virtual waiting area is introduced for each station. In this way, arrival passengers between any two consecutive trains have to wait at the virtual waiting areas until they are allowed to enter platforms and board the coming trains according to the control strategies.

So as to handle the time-varying passenger demands, the considered time period is equally divided into several intervals, in which the interval unit can be any value, such as $1 \mathrm{~min}$ and $30 \mathrm{~s}$ according to operational requirements $[13,14]$. The number of train services in the planning period can be predetermined properly by service providers. 


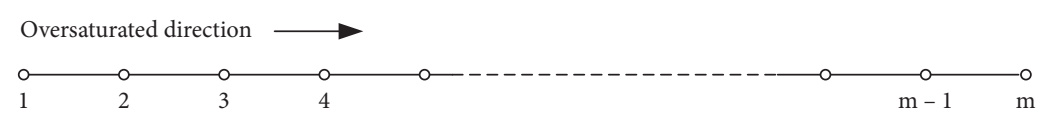

(a)

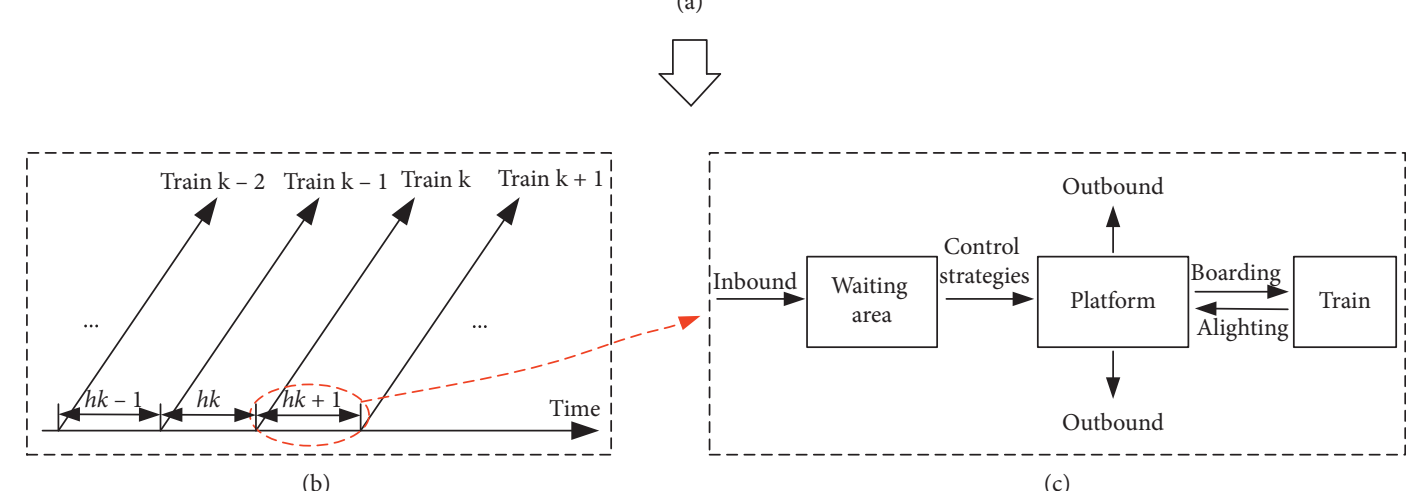

(b)

(c)

FIgURE 1: Urban rail line with heterogeneous headways and control strategies: (a) urban rail line; (b) train headways; (c) passenger flow control.

Additionally, for the sake of the Automatic Fare Collection (AFC) systems, the origin-destination passenger demands in each discrete time interval can be obtained from the previous travel data.

In brief, an integrated optimization model combining heterogeneous headways and passenger flow control strategies is formulated based on time-varying origin-destination passenger demands, aiming to rebalance the service of various stations and equilibrate the train loading capacity utilization.

3.1. Assumptions. Practically, train operations and passenger organizations are affected by many obscure factors. Hence, we first make the following assumptions throughout the current research.

Assumption 1. For simplicity, the optimization method is proposed for only one direction of a congested urban rail line, whose operations are not interfered by uncertainties. Every train stops at all stations, and the running time at any interstation segment and dwelling time at any station are pregiven. This assumption aims to make sure that the trains and passengers are organized as planned, which is common in timetabling studies [14].

Assumption 2. The time-varying number of arrival passengers for each station is pregiven. Besides, for any single station, the destination proportion of arrival passengers in each time interval is constant. This assumption is reasonable since the majority of trips in peak hours is commuting trip, and the residential locations and job places are relatively fixed.

Assumption 3. The total capacity of all train services provided can finally satisfy the total passenger demands in the studied time horizon. And all the involved passengers arrive at their individual origin stations before the last train departs.
Assumption 4. Since passenger flow control is implemented, every passenger will comply with the control arrangements and queue up at waiting areas. No stranded passengers will give up their trips or shift to other transit modes.

Assumption 5. Simplistically, the walking time from waiting areas to platforms will not be considered. The similar assumption can also be found in Shi et al. [31].

3.2. Notations. To facilitate the modeling process, definitions of sets, indices, parameters, and variables used in the formulation are summarized in Table 1.

\subsection{Constraints}

\subsubsection{Train Timetable Constraints}

(1) Headway. Commonly, overtaking is not permitted, and the operation sequence of all trains remains unchanged. To ensure operation safety and acceptable service quality, the headway between any two sequential trains should be restricted in a reasonable range. Note that the running time at any interstation segment and dwelling time at any station is fixed, and we only need to require the headway at the start terminal station to satisfy the minimum/maximum requirement $\left(H_{\min } / H_{\max }\right)$. Let $h_{k}$ denote the headway between train $k$ and train $k-1$ at the terminal station, and this constraint can be expressed as follows:

$$
H_{\min } \leq h_{k} \leq H_{\max }, \quad k \in \frac{K}{\{1\}} .
$$

Besides, the variation in two adjacent headways should not exceed the permitted value $H_{\text {diff }}$ for the smoothness of the timetable:

$$
-H_{\text {diff }} \leq h_{k}-h_{k-1} \leq H_{\text {diff }}, \quad k \in \frac{K}{\{1,2\}} .
$$


TABle 1: Notations used throughout the paper.

\begin{tabular}{|c|c|}
\hline $\begin{array}{l}\text { Sets } \\
S \\
K \\
T\end{array}$ & $\begin{array}{l}\text { Sets of stations indexed by } i, i^{\prime} ; S=\{1,2,3, \ldots, m\} \\
\text { Sets of train services indexed by } k, k^{\prime} ; K=\{1,2,3, \ldots, n\} \\
\text { Sets of discrete time intervals indexed by } t ; T=\{1,2,3, \ldots, p\}\end{array}$ \\
\hline $\begin{array}{l}\text { Parameters } \\
H_{\text {min }}, H_{\max } \\
H_{\text {diff }} \\
H_{\text {pfc }} \\
\mathrm{AF}, \mathrm{AL} \\
\mathrm{DW}_{i} \\
\mathrm{TR}_{i} \\
A_{i, t} \\
\mathrm{CI}_{i} \\
\rho_{i, i^{\prime}} \\
\mathrm{CT} \\
\mathrm{CP}_{i} \\
L_{i}^{\text {ave }} \\
F\left(k^{\prime}-k\right) \\
U\end{array}$ & $\begin{array}{l}\text { The minimum and maximum headways between two adjacent trains } \\
\text { The maximum variation for two adjacent headways } \\
\text { The headway threshold for implementing passenger flow control } \\
\text { Departure times at the start terminal station for the first and last trains } \\
\text { Dwelling time at station } i \\
\text { Running time from station } i-1 \text { to station } i \\
\text { Number of passengers arriving at station } i \text { in time interval } t \\
\text { Maximum capacity of facilities at station } i \text { for one-unit interval } \\
\text { Proportion of arrival passengers at station } i \text { whose destination is station } i^{\prime} \\
\text { Maximum train loading capacity } \\
\text { Maximum capacity of the platform at station } i \\
\text { Average train load factor of the segment between station iand station } i+1 \\
\text { Ahe penalty for passengers who miss } k^{\prime}-k \text { trains, which should be a monotone increasing function of } k^{\prime}-k \\
\text { A large positive number }\end{array}$ \\
\hline $\begin{array}{l}\text { Intermediate } \\
\text { variables } \\
d_{i, k} \\
b_{i, k, t} \\
q_{i, k}^{a} \\
\mu_{i, k, k^{\prime}} \\
q_{i, k}^{e} \\
q_{i, k}^{b} \\
q_{i, k}^{\text {al }} \\
q_{i, k}^{\text {on }} \\
\end{array}$ & $\begin{array}{l}\text { Departure time of train } k \text { at station } i \\
\text { Binary variable, which is } 1 \text { if time interval } t \text { is larger than the departure time of train } k-1 \text { at station } i \text { and no larger } \\
\text { than the departure time of train } k \text { at the same station and is } 0 \text { otherwise } \\
\text { Number of arrival passengers at station } i \text { between train } k-1 \text { and train } k \\
\text { Binary variable, which is } 1 \text { if all passengers arriving at station } i \text { between train } k-1 \text { and train } k \text { have entered the } \\
\text { platform when train } k^{\prime} \text { departs from the same station and is } 0 \text { otherwise } \\
\text { Number of passengers entering the platform at station } i \text { between train } k-1 \text { and train } k \\
\text { Number of passengers boarding train } k \text { at station } i \\
\text { Number of passengers alighting from train } k \text { at station } i \\
\text { Number of passengers on train } k \text { after departing from station } i\end{array}$ \\
\hline $\begin{array}{l}\text { Decis } \\
h_{k} \\
q_{i, k, k^{\prime}}\end{array}$ & $\begin{array}{l}\text { Headway between train } k-1 \text { and train } k \text { at the start terminal station } \\
\text { Number of passengers arriving at station } i \text { between train } k-1 \text { and train } k \text { who enter the platform between the } \\
\text { subsequent train } k^{\prime}-1 \text { and train } k^{\prime}\end{array}$ \\
\hline
\end{tabular}

(2) Last Train Timetable. In this study, the departure times of the first and last trains at the start terminal station are pregiven. This constraint can be characterized as follows:

$$
\mathrm{AF}+\sum_{k=2}^{n} h_{k}=\mathrm{AL}
$$

(3) Departure Time. Note that the interstation segment running time $\mathrm{TR}_{i^{\prime}}$ and station dwelling time $\mathrm{DW}_{i^{\prime}}$ are pregiven constants, and the departure time for each train at each station $d_{i, k}$ can be calculated through the headways at the start terminal station:

$$
d_{i, k}=\mathrm{AF}+\sum_{k^{\prime}=2}^{k} h_{k^{\prime}}+\sum_{i^{\prime}=2}^{i}\left(\mathrm{DW}_{i^{\prime}}+\mathrm{TR}_{i^{\prime}}\right), \quad i \in S, k \in K .
$$

(4) Effective Arrival Time Period. Furthermore, to facilitate evaluating the number of arrival passengers at each station between any two adjacent trains, a binary variable $b_{i, k, t}$ is intermediately introduced to identify whether time interval $t$ is between train $k-1$ and train $k$ at station $i$.

As shown in Figure 2, each train departs from station $i$ at the end of one discrete time interval, and the effective time period for passengers arriving at station $i$ between train $k-1$ and train $k$ can be denoted as $\left(d_{i, k-1}, d_{i, k}\right]$. If $t \in\left(d_{i, k-1}, d_{i, k}\right]$, then $b_{i, k, t}=1$; otherwise, $b_{i, k, t}=0$. The effective arrival time period can be formulated as follows:

$$
\begin{gathered}
b_{i, k, t}<1+\frac{t-d_{i, k-1}}{U}, \quad i \in S, k \in K, t \in T . \\
b_{i, k, t} \leq 1-\frac{t-d_{i, k}}{U}, \quad i \in S, k \in K, t \in T, \\
\sum_{t=1}^{p} b_{i, k, t}=d_{i, k}-d_{i, k-1}, \quad i \in S, k \in K .
\end{gathered}
$$

Equation (5) describes that time intervals less than or equal to the departure time of train $k-1$ are infeasible for passengers arriving between train $k-1$ and train $k$. Note that $d_{i, k-1}$ is set as zero if $k$ is equal to 1 . Similarly, Equation (6) identifies that time intervals greater than the departure time 


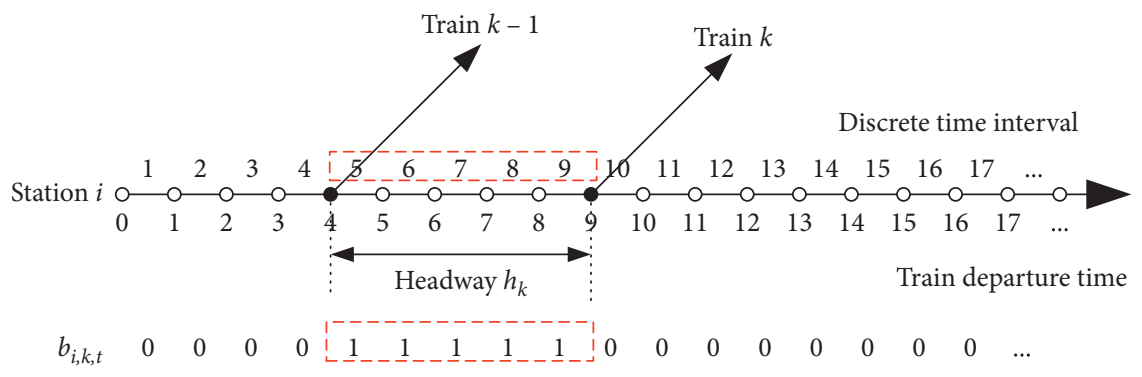

FIGURE 2: Illustration of train operation.

of train $k$ is inefficient. Equation (7) guarantees that $b_{i, k, t}$ is equal to 1 when the time interval $t$ belongs to $\left(d_{i, k-1}, d_{i, k}\right]$.

\subsubsection{Passenger Flow Control Constraints}

(1) Arrival Passengers. Based on the effective arrival time period for passengers at each station, the total number of passengers arriving at station $i$ between train $k-1$ and train $k$, denoted by $q_{i, k}^{a}$, can be calculated as follows. Note that $A_{i, t}$ denotes the pregiven passenger arrival volume at station $i$ and time interval $t$ :

$$
q_{i, k}^{a}=\sum_{t=1}^{p} A_{i, t} b_{i, k, t}, \quad i \in S, k \in K
$$

(2) Passengers Entering Platforms. As discussed above, once passenger flow control strategies are implemented, and arrival passengers are required to queue up at waiting areas until they are allowed to enter platforms consecutively. The passenger flow control process is illustrated in Figure 3, in which the time horizon is divided into several control periods. For description convenience, control period $k$ represents the time range between train $k-1$ and train $k$. Therefore, the control variables, denoted by $q_{i, k, k^{\prime}}$, are the decision objects in this paper, which means the number of passengers who arrive at station $i$ in control period $k$ and enter the platform in the subsequent control period $k^{\prime}$. And $\mu_{i, k, k^{\prime}}$ denotes an intermediate variable that decides whether all passengers arriving at station $i$ between train $k-1$ and train $k$ have entered the platform when train $k^{\prime}$ departs from the same station:

$$
-\frac{q_{i, k}^{a}-\sum_{x=k}^{k^{\prime}} q_{i, k, x}}{U}<\mu_{i, k, k^{\prime}} \leq 1-\frac{q_{i, k}^{a}-\sum_{x=k}^{k^{\prime}} q_{i, k, x}}{U}, \quad i \in S, k \leq k^{\prime} \in K,
$$

$$
\begin{aligned}
q_{i, k, k^{\prime}} & \leq U \mu_{i, k-1, k^{\prime}}, \quad i \in S, k-1, k \leq k^{\prime} \in K, \\
\frac{h_{k}-H_{p f c}}{U}<\mu_{i, k, k^{\prime}}, \quad i \in S, k \leq k^{\prime} \in K, & \\
\sum_{k^{\prime}=k}^{n} q_{i, k, k^{\prime}} & =q_{i, k}^{a}, \quad i \in S, k \in K,
\end{aligned}
$$

$$
q_{i, k}^{e}=\sum_{k^{\prime}=1}^{k} q_{i, k^{\prime}, k}, \quad i \in S, k \in K .
$$

Specifically, equations (9) and (10) describe the temporal FIFO principle, in which passengers arriving early have the absolute priority to enter the platform at the same station. Equation (9) identifies whether passengers arriving at station $i$ in control period $k$ have already enter the platform completely when it is in the subsequent control period $k^{\prime}$. That is, if $q_{i, k}^{a}=\sum_{x=k}^{k^{\prime}} q_{i, k, x}$, then $\mu_{i, k, k^{\prime}}=1$; otherwise, $\mu_{i, k, k^{\prime}}=0$. On this basis, Equation (10) guarantees that passengers arriving at station $i$ cannot enter the platform before those who arrive earlier.

Equation (11) identifies the time to implement passenger flow control strategies. When the designed headways exceed the threshold $H_{\mathrm{pfc}}$, it reflects that the current train loading capacity can accommodate the passenger demands already. Thus, the passenger flow control is unnecessary, and $\mu_{i, k, k^{\prime}}$ is equal to 1 .

Equation (12) indicates that the whole passengers involved in considered time horizon at every station will be served eventually.

Equation (13) evaluates the total passengers allowed to enter the platform at station $i$ in control period $k$, which is denoted by $q_{i, k}^{e}$.

(3) Facility Capacity. Realistically, the number of passengers permitted to enter platforms at waiting areas is limited by entrance facilities, which means $q_{i, k}^{e}$ should be bounded by the corresponding passing capacity. Note that $\mathrm{CI}_{i}$ is the maximum capacity of entry facilities at station $i$ within one unit interval, and $h_{k}$ is set as $H_{\min }$ when $k$ is equal to 1 :

$$
q_{i, k}^{e} \leq \mathrm{CI}_{i} h_{k}, \quad i \in S, k \in K \text {. }
$$

\subsubsection{Dynamic Passenger Loading Constraints}

(1) Boarding Passengers. The vital goals of adopting collaborative passenger flow control are to prevent platforms from high passenger accumulations and guarantee the safety of passengers. It is desirable that the remaining train loading capacity can satisfy all the passengers stranded at platforms. To this end, the control strategies optimized in this study require that all passengers on platforms can get on the first coming train. Thus, the number of passengers boarding train $k$ at station $i$, denoted by $q_{i, k}^{b}$, can be expressed as follows: 


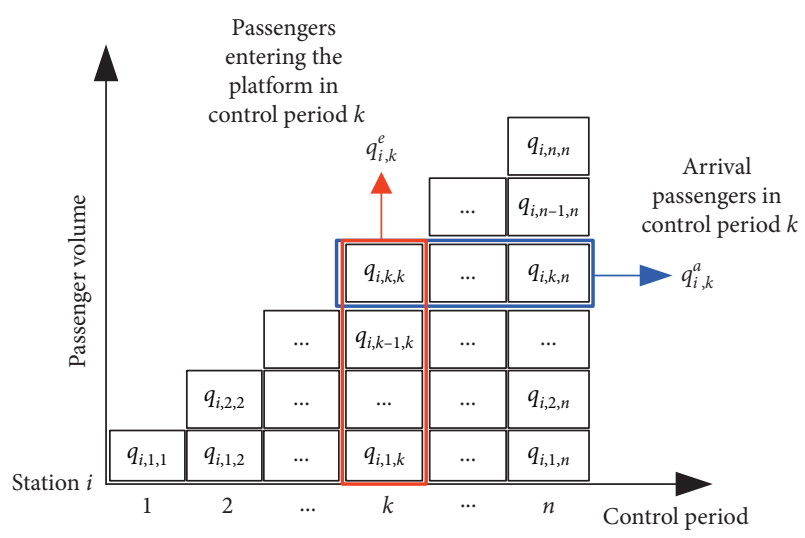

Figure 3: Illustration of the passenger flow control process.

$$
q_{i, k}^{b}=q_{i, k}^{e}, \quad i \in S, k \in K
$$

(2) Alighting Passengers. As assumed above, the destination proportion of passengers at one specific station is fixed, and then, the number of passengers alighting from train $k$ at station $i$, denoted by $q_{i, k}^{a l}$, can be calculated according to the number of boarding passengers at upstream stations. $\rho_{i^{\prime}, i}$ is the predetermined proportion of passengers who take a train from station $i^{\prime}$ to station $i$ :

$$
q_{i, k}^{a l}=\sum_{i^{\prime}=1}^{i-1} \rho_{i^{\prime}, i} q_{i^{\prime}, k}^{b}, \quad i \in S, k \in K .
$$

(3) Train Capacity. The variation in passengers in each train at each interstation segment depends on the boarding and alighting process dynamically. In addition, the maximum number of passengers on train is limited by the train loading capacity. Then, the number of passengers on each train after departing from each station, denoted by $q_{i, k}^{\text {on }}$, can be characterized in equations (17) and (18). CT is the maximum loading capacity for one train:

$$
\begin{aligned}
& q_{i, k}^{\text {on }}=\left\{\begin{array}{ll}
q_{i, k}^{b}, & \text { if } i=1, \\
q_{i-1, k}^{\text {on }}+q_{i, k}^{b}-q_{i, k}^{a l}, & \text { if } 2 \leq i \leq m,
\end{array} \quad k \in K,\right. \\
& q_{i, k}^{\text {on }} \leq C T, \quad i \in S, k \in K .
\end{aligned}
$$

(4) Platform Capacity. Since all the passengers at platforms will give way to the alighting passengers first and then get on board. The maximum assembling at platforms occurs at the end of alighting process and should not exceed the platform capacity $\mathrm{CP}_{i}$ :

$$
q_{i, k}^{b}+q_{i, k}^{\mathrm{al}} \leq \mathrm{CP}_{i}, \quad i \in S, k \in K
$$

\subsection{Objective Function}

(1) Equity Performance. As a key component of public transportation, urban rail transit should provide equitable services to any individual or group. Specifically, the number of missed trains for every passenger is supposed to be equal [16]. However, some passengers waiting at relatively downstream stations always suffer more missed trains in congested situations; as a result, service imbalance problem occurs and leads to low service quality. In this study, an imbalance indicator $E$ is introduced to describe the overall equity performance of an oversaturated urban rail line, which is calculated as a penalty value of the number of missed trains, shown as follows:

$$
E=\frac{\sum_{i=1}^{m} \sum_{k=1}^{n} \sum_{k^{\prime}=k}^{n}\left(F\left(k^{\prime}-k\right) q_{i, k, k^{\prime}}\right)}{\sum_{i=1}^{m} \sum_{t=1}^{p} A_{i, t}},
$$

where, in Equation (18), $k^{\prime}-k$ means the number of trains missed by passengers who arrive at their origin stations in control period $k$ and enter platforms in control period $k^{\prime}$. Obviously, $k^{\prime}$ is greater than or equal to $k$. The function $F\left(k^{\prime}-k\right)$ is essentially a parameter that denotes the penalty for passengers missing $k^{\prime}-k$ trains. For passengers who miss more trains, the penalty is expected to be larger. Consequently, the larger the number of missed trains $k^{\prime}-k$ is, the larger the value of $F\left(k^{\prime}-k\right)$ is. Based on the above principle, $F\left(k^{\prime}-k\right)$ could be a monotone increasing function of the number of missed trains $k^{\prime}-k$. In this study, we set $F\left(k^{\prime}-k\right)=\left(k^{\prime}-k\right)^{2}$ subjectively. The similar practice of parameter choice can be seen in [16] as well.

(2) Train Load Factor. In addition to equity performance, the train load factor is another crucial indicator related to the train timetabling. Typically, urban rail operators want to reduce the average train load factor and balance the loading capacity utilization for each train under oversaturated circumstances. Since the number of train services and travel demands involved in the studied time horizon is predetermined, the average train load factor at each segment, denoted by $L_{i}^{\text {ave }}$, is irreducible and can be calculated in advance. Therefore, this study only considers the load factor equilibrium $L$ for each train at each segment, which is described as follows:

$$
\begin{aligned}
-L_{i, k} & \leq \frac{q_{i, k}^{\text {on }}}{\mathrm{CT}}-L_{i}^{\mathrm{ave}} \leq L_{i, k}, \quad i \in S, k \in K, \\
L & =\sum_{i=1}^{m-1} \sum_{k=1}^{n} L_{i, k} .
\end{aligned}
$$

Equation (21) identifies $L_{i, k}$ as the absolute difference between the load factor for each train and the average value at the same segment, and Equation (22) describes the sum of absolute differences. Note that the absolute value is linearized from the initial expression $L_{i, k}=\left|q_{i, k}^{\text {on }} / \mathrm{CT}-L_{i}^{\text {ave }}\right|$ in constraint (21), as the aim of the method is to minimize train load factor equilibrium indicator $L$ in constraint (22).

3.5. Model. As all the constraints and objective functions have been established linearly, the mixed-integer linear programming model with respect to the train timetabling 
and passenger flow control (simplified as TPFC model) is formulated as follows:

$$
\begin{cases}\min & E, \\ \min & L, \\ \text { constraints } & (1)-(22), \\ h_{k} \in N, & k \in K .\end{cases}
$$

\section{Solution Algorithm}

The formulated optimization model turns out to be a multiobjective mixed-integer linear programming model, which is difficult to accurately find the optimal solution. Thus, the following discussion aims to develop a heuristic algorithm to obtain an approximate optimal solution efficiently. We first transfer the multiobjective problem into an equivalent single-objective problem; then, an iterative heuristic method is designed, and detailed techniques are introduced.

(1) Single-Objective Transformation. The linear weighting method is a common and simple approach to solve multiobjective problems [35]. In this study, weighting coefficients $\omega_{1}$ and $\omega_{2}$ are introduced for the imbalance indicator $E$ and train load factor equilibrium indicator $L$, respectively; thus, the original multiobjective functions are as follows:

$$
\min Z=\omega_{1} E+\omega_{2} L .
$$

Typically, when we set $\omega_{1}=1$, then $\omega_{2}=E_{\mathrm{NPFC}} / L_{\mathrm{NPFC}}$. Here, $E_{\mathrm{NPFC}}$ and $L_{\mathrm{NPFC}}$ are values of the imbalance indicator and train load factor equilibrium indicator under the scenario with respect to the original timetable and noncollaborative passenger flow control (simplified as NPFC model).

(2) The Iterative Heuristic Method. As for the appropriate solution method, the complexity of the proposed model is discussed first. Clearly, the scale of TPFC model depends on the numbers of stations, train services, and interval timestamps, and crucial variables and constraints are listed in Table 2. Intuitively, we consider an urban rail line with 10 stations and 40 train services, and the time horizon is divided into 100 timestamps. In this case, the model consists of 8240 decision variables and more than 100,000 constraints. As expect, it is obvious that the scale of the proposed TPFC model is larger for real-world cases. As a consequence, the existing accurate optimization methods are hard to deal with this integrated problem.

Theoretically, an effective method to address such largescale optimization model is decomposing it into several tractable small-scale subproblems, which can be solved by accurate or heuristic methods. Note that the binary variables $b_{i, k, t}$ develop the connection between the train timetabling and passenger flow control and account for a large proportion of the problem complexity. If we predetermine the headways of all the trains, variables $b_{i, k, t}$ can be evaluated
TABLE 2: Numbers of crucial variables and constraints in the model.

\begin{tabular}{lc}
\hline Variables or constraints & Dimension \\
\hline Decision variables & $|K|$ \\
$h_{k}$ & $|S| \cdot(|K|+1) \cdot|K| / 2$ \\
$q_{i, k, k^{\prime}}$ & \\
\hline Crucial constraints & $|K|-1$ \\
Constraint (1) & $|K|-2$ \\
Constraint (2) & 1 \\
Constraints (3), (20), (22) & $|S| \cdot|K|$ \\
Constraints (4), (7), (8), (12)-(19), (21) & $|S| \cdot|K| \cdot|T|$ \\
Constraints (5)-(6) & $|S| \cdot(|K|+1) \cdot|K|$ \\
Constraint (9) & $|S| \cdot(|K|+1) \cdot|K| / 2$ \\
Constraints (10)-(11)
\end{tabular}

easily in advance. Then, the remainder is a passenger flow control problem with a scheduled timetable, which consists of a relatively small-scale variables and constraints and can be solved efficiently by commercial optimization solvers (i.e., Gurobi). In this paper, the original integrated problem is decomposed into train timetabling subproblem and passenger flow control subproblem. The train timetabling subproblem is solved by the tabu search method to obtain feasible train headways, and the results of the former subproblem are taken as inputs for the passenger flow control subproblem. We then optimize the passenger flow control strategies by Gurobi solver, and its optimal objective value will be an assessment index to guide the search process in the next iteration. The specific iterative heuristic search procedure is summarized as Algorithm 1.

Some notations in the algorithm are identified in the following discussion. $x$ denotes the iteration index, while $X_{\max }$ is the maximum number of iterations. $y$ denotes the index that the current best solution has not updated, while $Y_{\max }$ is the maximum limitation. The timetable is denoted by a headway vector $H=\left(h_{1}, h_{2}, h_{3}, \ldots, h_{n-1}, h_{n}\right)$ for all the consecutive trains; specifically, $H^{x, 0}$ denotes the seed solution for iteration $x$, and $H^{*}$ denotes the current best solution. $H_{\mathrm{NS}}^{x}=\left\{H^{x, c} \mid c=1,2, \ldots, n_{\mathrm{NS}}-1, n_{\mathrm{NS}}\right\}$ denotes the set of neighbor solutions for iteration $x$, in which $n_{\mathrm{NS}}$ is the number of neighbors. TL denotes the tabu list, whose length is $n_{\mathrm{TL}} . Z^{x}$ denotes the best objective value for iteration $x$, and $Z^{*}$ denotes the current best objective value.

\section{Case Study}

In this section, the proposed approach is applied to a simple case and a real-world case of Beijing Subway Batong Line. The simple case is to verify the efficiency of the proposed algorithm in comparison to using the Gurobi solver directly through several different-scale instances. Furthermore, the real-world case based on Beijing Subway Batong Line is implemented to demonstrate performances of the proposed integrated method combining the train timetabling and collaborative passenger flow control.

5.1. Simple Example. A simple numerical case with five stations, named by A, B, C, D and E, is assumed to compare the proposed algorithm to use the Gurobi solver directly. The 
Step 1: Initiation. Set $x=0, y=0$, and TL $=$ null. Input the original seed solution $H^{x, 0}$ and then use the Gurobi solver to optimize the passenger flow control strategies and obtain corresponding objective value $Z^{x}$. Let $H^{*}=H^{x, 0}, Z^{*}=Z^{x}$, and $H^{x+1,0}=H^{x, 0}$. Update $x=1$ and go to step 2 .

Step 2: generate neighbor set $H_{\mathrm{NS}}^{x}$ iteratively as follows (initially set $c=1$ ):

(1) Duplicate $H^{x, 0}$ as $H^{x, c}$ and then select two elements $h 1^{x, c}$ and $h 2^{x, c}$ in $H^{x, c}$ and generate a random variation $\Delta h \in\left[1, H_{\text {diff }}\right]$; then, let $h 1^{x, c}=h 1^{x, c}-\Delta h$ and $h 2^{x, c}=h 2^{x, c}+\Delta h$.

(2) If $H^{x, c}$ satisfies headway constraints Equations (1) and (2), then optimize the control strategies and obtain the corresponding objective value; insert $H^{x, c}$ into $H_{\mathrm{NS}}^{x}$ and set $c=c+1$.

(3) If $c>n_{\mathrm{NS}}$, then go to step 3; otherwise, continue this iteration.

Step 3: obtain the best solution $H^{x}$ from $H_{\mathrm{NS}}^{x}$ and its corresponding objective value $Z^{x}$. If $Z^{x}<Z^{*}$, then let $H^{*}=H^{x}, Z^{*}=Z^{x}$, $x=x+1$, and $y=0$, update the next iteration seed $H^{x, 0}=H^{x}$, and insert $H^{x, 0}$ into TL; otherwise, obtain the best solution $H^{x^{\prime}}$ from $H_{\mathrm{NS}}^{x}$ that is not in TL, and let $x=x+1, y=y+1$, and $H^{x, 0}=H^{x^{\prime}}$; insert $H^{x, 0}$ into TL. Go to step 4 .

Step 4: if $x \leq X_{\max }$ and $y \leq Y_{\max }$, go to step 2; otherwise, output the optimal timetable $H^{*}$ and corresponding passenger flow control strategies and objective value and stop.

Algorithm 1: An iterative heuristic algorithm to solve the proposed model.

planning timetable horizon is one hour, in which the first and last trains depart the start terminal station at 3 min and $48 \mathrm{~min}$, respectively. The running time at each interstation segment is $150 \mathrm{~s}$, and the dwelling time at each station is $30 \mathrm{~s}$. Moreover, the minimum and maximum headways are $2 \mathrm{~min}$ and $6 \mathrm{~min}$, respectively, and the allowed maximum headway variation is set as $1 \mathrm{~min}$. When the headway exceeds $5 \mathrm{~min}$, passenger flow control is not performed. Besides, the train capacity and platform capacity are set to be 500 and 900 , respectively, and the entrance facilities of each station allow 270 persons to enter the platform per minute. In addition, the total passenger demands, time range, and destination rate for arrival passengers at each station are shown in Table 3; meanwhile, arrival passengers at each station obey the distribution illustrated in Figure 4. The values of other parameters are $X_{\text {max }}=50, \quad Y_{\text {max }}=10, \quad n_{\mathrm{NS}}=45$, and $n_{\mathrm{TL}}=10$. Note that the values of $E_{\mathrm{NPFC}}$ and $L_{\mathrm{NPFC}}$ are evaluated from the original timetables with equal headways.

In this simple case, the numbers of train services and timestamps are considered to generate different instances. For description convenience, we use I-T-K to represent the instance, in which $T$ denotes the number of timestamps and $\mathrm{K}$ denotes the number of train services. For example, I-120-10 represents that the one hour horizon is divided into 120 timestamps and 10 train services are provided. In each instance, all the parameters are the same except that the total passenger demands are adjusted in proportion to the total train capacity. All instances are computed on a personal computer with an Intel Core i5, $1.60 \mathrm{HGz} \mathrm{CPU}$, and $8 \mathrm{~GB}$ RAM. The version of Gurobi solver is Academic 9.0, and corresponding options are default except that time limit is set as 3600 s.

Table 4 shows the computational results of the proposed "TS + Gurobi" algorithm and Gurobi, including the objective value, CPU time, and optimality gap between two methods. It can be found that the CPU time for Gurobi increases a lot when the number of timestamps and trains increase, while the proposed algorithm can obtain near-optimal results with a fairly short time. Particularly, in the instances with more than 180 timestamps and 13 train services, the Gurobi solver cannot generate objective values with a MIPGap less than
$30 \%$ in one hour, while the results obtained by the proposed algorithm are significantly more optimal, resulting in negative gaps. Furthermore, Figure 5 plots the tendency of the best objective values of each iteration for instance I-240-13. It is clear that the proposed algorithm iteratively decreases the objective value, and the best solution is achieved after 13 iterations. In iterations 1 to 6 , the encountered objective value is reduced significantly, while in iterations 7 to 13 , the objective value decreases with a relatively flat tendency. The above results imply that the proposed algorithm combining the tabu search and Gurobi solver can solve the integrated problem with a high efficiency.

\subsection{Practical Experiments on Beijing Subway Batong Line}

5.2.1. Basic Data. Beijing Subway Batong Line is $18.94 \mathrm{~km}$ and comprises 13 stations. It originates from TQ Station to SH Station, linking the residential zone in Tongzhou District and Beijing central business zone of Guomao, shown in Figure 6. SH and SHD Stations are transfer stations connecting to Beijing Subway Line 1, and a great many of commuters boarding the trains in the TQ $\longrightarrow \mathrm{SH}$ direction get off here or transfer to other stations.

The direction from TQ Station to SH Station is the focus in this case study, and the morning peak hours from 7:00 to 10:00 on a typical Thursday are selected as the considered time horizon. The unit discrete time interval is set as 30 seconds; thus, the time horizon is divided into 360 timestamps. In the study period in Batong Line, the original headway at TQ Station is $3 \mathrm{~min}, 4 \mathrm{~min}$, and $5 \mathrm{~min}$, respectively, for 7:00 8:20, 8:20 9:00, and 9:00 9:30. Thus, the total number of 41 train services is considered, and the first and last trains depart TQ Station at 7:05 and 9:25, respectively. The rated loading capacity for each train is 1460 , and the maximum load factor should not exceed $130 \%$, resulting in a maximum train capacity of 1898 . The capacity of platforms for transfer stations SH and SHD is 950, and that for other stations is 850. Additionally, the dwelling time and running time are displayed in Table 5. As for optimization, the minimum and maximum headways are $2 \mathrm{~min}$ and $6 \mathrm{~min}$, respectively, and the allowed maximum headway variation 
TABLE 3: Characteristics of passenger flow for each station.

\begin{tabular}{|c|c|c|c|c|c|c|c|}
\hline \multirow{2}{*}{ Stations } & \multirow{2}{*}{ Passenger demands* (person) } & \multirow{2}{*}{ Time range $\left[t_{s}, t_{e}\right]$} & \multicolumn{5}{|c|}{ Destination rate } \\
\hline & & & $\mathrm{A}$ & B & $\mathrm{C}$ & $\mathrm{D}$ & $\mathrm{E}$ \\
\hline A & 1790 & {$[0,48]$} & 0.00 & 0.12 & 0.20 & 0.24 & 0.44 \\
\hline $\mathrm{B}$ & 1070 & {$[3,51]$} & & 0.00 & 0.13 & 0.33 & 0.54 \\
\hline $\mathrm{C}$ & 1570 & {$[6,54]$} & & & 0.00 & 0.59 & 0.41 \\
\hline $\mathrm{D}$ & 930 & {$[9,57]$} & & & & 0.00 & 1.00 \\
\hline $\mathrm{E}$ & 0 & {$[12,60]$} & & & & & 0.00 \\
\hline
\end{tabular}

${ }^{*}$ Passenger demands are for instances with 10 train services; $t_{s}$ and $t_{e}$ denote the start and end arrival times for passengers involved in the study, unit: min.

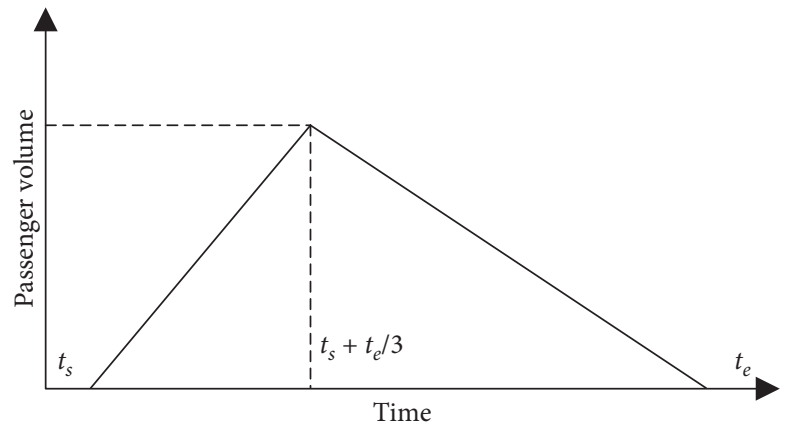

Figure 4: Temporal distribution of arrival passengers.

TABle 4: Performance comparison of Gurobi and TS + Gurobi

\begin{tabular}{|c|c|c|c|c|}
\hline Instances & Method & Objective & CPU time (s) & Gap (\%) \\
\hline \multirow{2}{*}{ I-60-10 } & Gurobi & 0.4954 & 2.3 & - \\
\hline & TS + Gurobi & 0.4954 & 36.0 & 0.00 \\
\hline \multirow{2}{*}{ I-60-16 } & Gurobi & 0.5174 & 59.5 & - \\
\hline & TS + Gurobi & 0.5196 & 142.1 & 0.43 \\
\hline \multirow{2}{*}{ I- $120-10$} & Gurobi & 0.4721 & 30.4 & - \\
\hline & TS + Gurobi & 0.4724 & 43.9 & 0.06 \\
\hline \multirow{2}{*}{ I-120-16 } & Gurobi & 0.4230 & 2300.3 & - \\
\hline & TS + Gurobi & 0.4233 & 247.3 & 0.07 \\
\hline \multirow{2}{*}{ I-180-13 } & Gurobi & 0.3578 & $3600 / 36.2 \%^{*}$ & - \\
\hline & TS + Gurobi & 0.3330 & 76.5 & -6.93 \\
\hline \multirow{2}{*}{ I-240-13 } & Gurobi & 0.4596 & $3600 / 44.4 \%^{*}$ & - \\
\hline & TS + Gurobi & 0.3935 & 99.4 & -14.38 \\
\hline \multirow{2}{*}{ I-240-16 } & Gurobi & 0.5617 & $3600 / 54.2 \%^{*}$ & - \\
\hline & TS + Gurobi & 0.3882 & 192.7 & -30.89 \\
\hline \multirow{2}{*}{ I-240-19 } & Gurobi & 0.8901 & $3600 / 45.9 \% *$ & - \\
\hline & TS + Gurobi & 0.7809 & 442.7 & -12.27 \\
\hline
\end{tabular}

The value with * is the MIPGap of Gurobi solver in a given time limit $3600 \mathrm{~s}$. MIPGap is an internal index of the Gurobi solver that evaluates the relative difference of the current solution with the possible optimal solution. The gap is calculated by (Objective $\left.\left.\mathrm{TS}+\mathrm{Gurobi}-\mathrm{Objective}_{\mathrm{Gurobi}}\right) / \mathrm{Objective}_{\mathrm{Gurobi}}\right)$.

between any two adjacent trains is limited within $1 \mathrm{~min}$. Besides, passenger flow control is not applied when the headways exceed $5 \mathrm{~min}$.

It is assumed that a virtual train departing from TQ Station at 7:02 will take all the stranded passengers away at each station; therefore, the passengers involved in this case study are those arriving at each station between the departure times of the virtual train and the last practical train. Specifically, the time range for arrival passengers and destination rate at each station are pregiven in $\mathrm{Ta}-$ ble 6. TQ Station and CMDX Station are taken as examples to illustrate time-varying arrival passengers, shown in Figure 7.

In the iterative heuristic algorithm, we set $X_{\max }=80$, $Y_{\max }=10, n_{N S}=60$, and $n_{T L}=10$. The passenger flow control strategies are solved by Gurobi 9.0 Academic Version with the MIPGap $0.1 \%$ and time limit $5 \mathrm{~min}$.

5.2.2. Optimization Results. In the experiment for Beijing Subway Batong Line, we first evaluate the service performance considering the original timetable and 


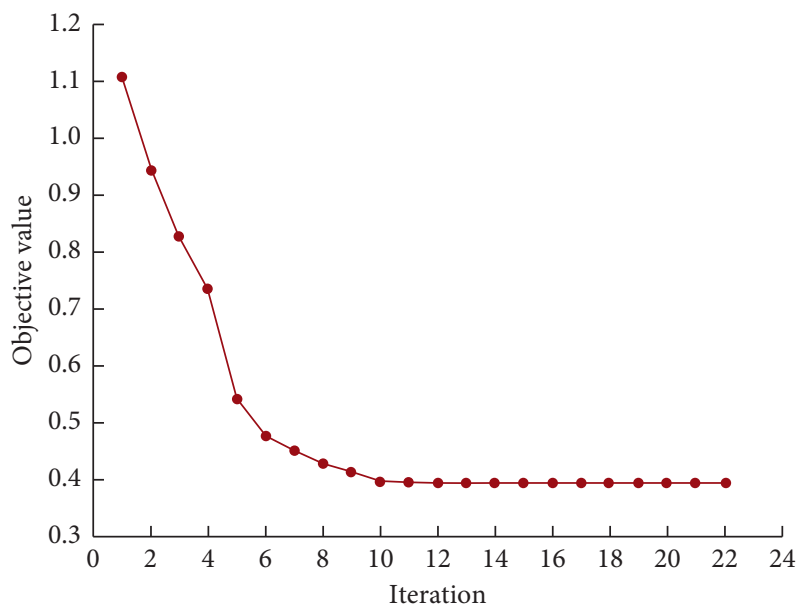

FIgURE 5: Variation tendency of the best objective value for instance I-240-13.
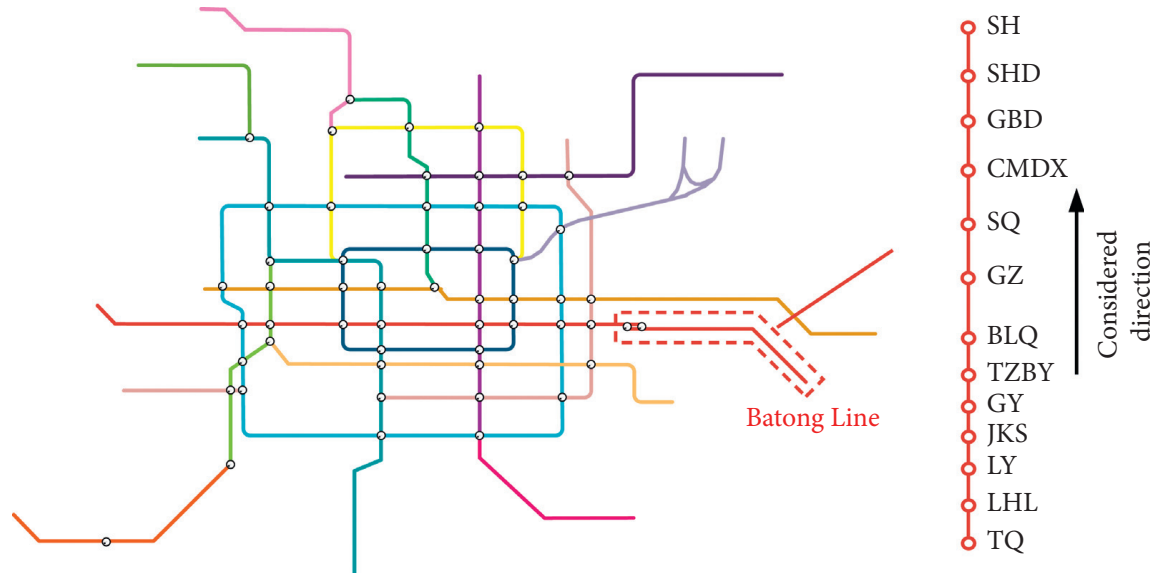

Figure 6: Batong Line in Beijing subway network.

TABLE 5: Dwelling time and running time.

\begin{tabular}{lccc}
\hline Stations & Dwelling time $(\mathrm{s})$ & Segments & Running time $(\mathrm{s})$ \\
\hline TQ & 45 & TQ $\longrightarrow$ LHL & 75 \\
LHL & 30 & LHL $\longrightarrow$ LY & 105 \\
LY & 30 & LY $\longrightarrow$ JKS & 105 \\
JKS & 30 & JKS $\longrightarrow$ GY & 90 \\
GY & 30 & GY $\longrightarrow$ TZBY & 120 \\
TZBY & 45 & TZBY $\longrightarrow$ BLQ & 135 \\
BLQ & 30 & BLQ $\longrightarrow$ GZ & 150 \\
GZ & 45 & GZ $\longrightarrow$ SQ & 135 \\
SQ & 45 & SQ $\longrightarrow$ CMDX & 135 \\
CMDX & 45 & CMDX $\longrightarrow$ GBD & 135 \\
GBD & 30 & GBD $\longrightarrow$ SHD & 120 \\
SHD & 30 & SHD $\longrightarrow$ SH & 135 \\
SH & 45 & & \\
\hline
\end{tabular}

noncollaborative passenger flow control strategies (denoted by the NPFC model). In the NPFC model, arrival passengers are allowed to enter platforms as long as the coming trains have sufficient loading capacity. Consequently, the values of the imbalance indicator and train load factor equilibrium indicator turn out to be 7.07 and 63.4, respectively. The timetable and resulted passenger accumulation situation are illustrated in Figure 8. Without collaborative passenger flow control, passenger accumulation situations at CMDX and SQ Stations are extremely unbalanced. In particular, the 
TABLE 6: Time range and destination rate for arrival passengers at each station.

\begin{tabular}{|c|c|c|c|c|c|c|c|c|c|c|c|c|c|}
\hline \multirow{2}{*}{ Stations } & \multirow{2}{*}{ Time range } & \multicolumn{12}{|c|}{ Destination rate (\%) } \\
\hline & & 2 & 3 & 4 & 5 & 6 & 7 & 8 & 9 & 10 & 11 & 12 & 13 \\
\hline TQ & 7:02 9:24 & 0 & 0 & 0.05 & 0 & 2.81 & 0 & 1.15 & 3.21 & 1.82 & 5.33 & 41.98 & 43.65 \\
\hline LHL & 7:03 9:25 & & 0 & 0 & 0 & 3.41 & 0 & 0.42 & 3.14 & 2.09 & 4.48 & 43.08 & 43.39 \\
\hline LY & 7:06 9:28 & & & 0 & 0 & 1.97 & 0 & 1.00 & 3.04 & 1.90 & 3.99 & 43.79 & 44.31 \\
\hline JKS & 7:08 9:30 & & & & 0 & 1.49 & 0 & 0.45 & 2.35 & 1.90 & 4.48 & 45.05 & 44.28 \\
\hline GY & 7:10 9:32 & & & & & 0 & 0 & 0.82 & 2.08 & 1.94 & 4.40 & 45.73 & 45.04 \\
\hline TZBY & 7:13 9:35 & & & & & & 0 & 0.57 & 3.53 & 2.41 & 6.31 & 46.16 & 41.02 \\
\hline BLQ & 7:15 9:37 & & & & & & & 0 & 0.91 & 1.14 & 2.86 & 47.47 & 47.61 \\
\hline GZ & 7:19 9:41 & & & & & & & & 0.18 & 0.41 & 2.95 & 49.81 & 46.65 \\
\hline SQ & 7:22 9:44 & & & & & & & & & 0 & 0.75 & 49.47 & 49.78 \\
\hline CMDX & 7:25 9:47 & & & & & & & & & & 0 & 50.15 & 49.85 \\
\hline GBD & 7:27 9:49 & & & & & & & & & & & 54.23 & 45.77 \\
\hline SHD & 7:30 9:52 & & & & & & & & & & & & 100 \\
\hline
\end{tabular}

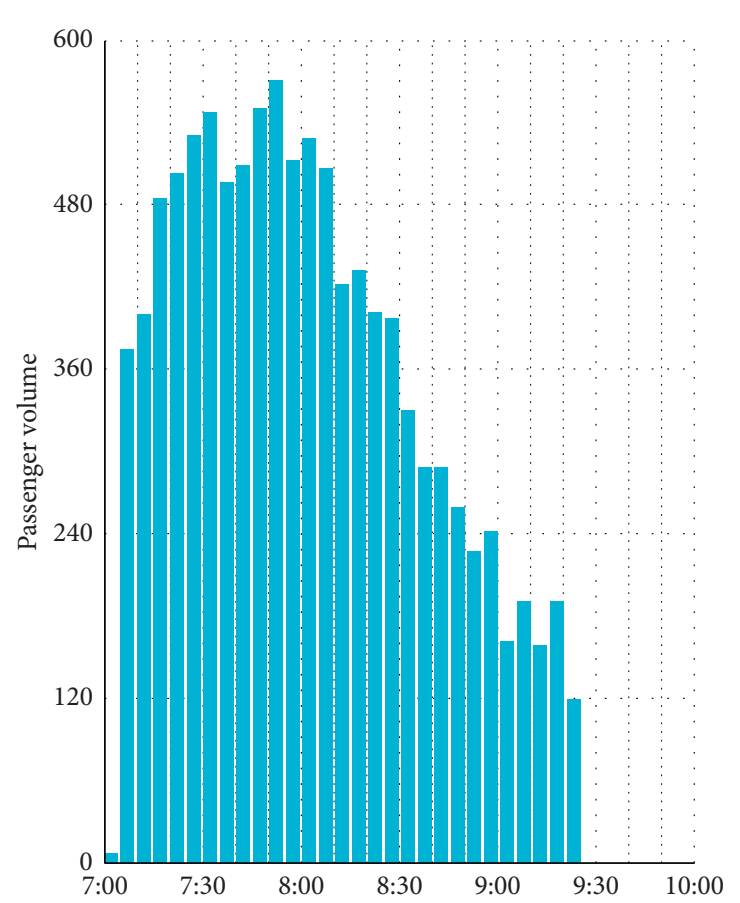

(a)

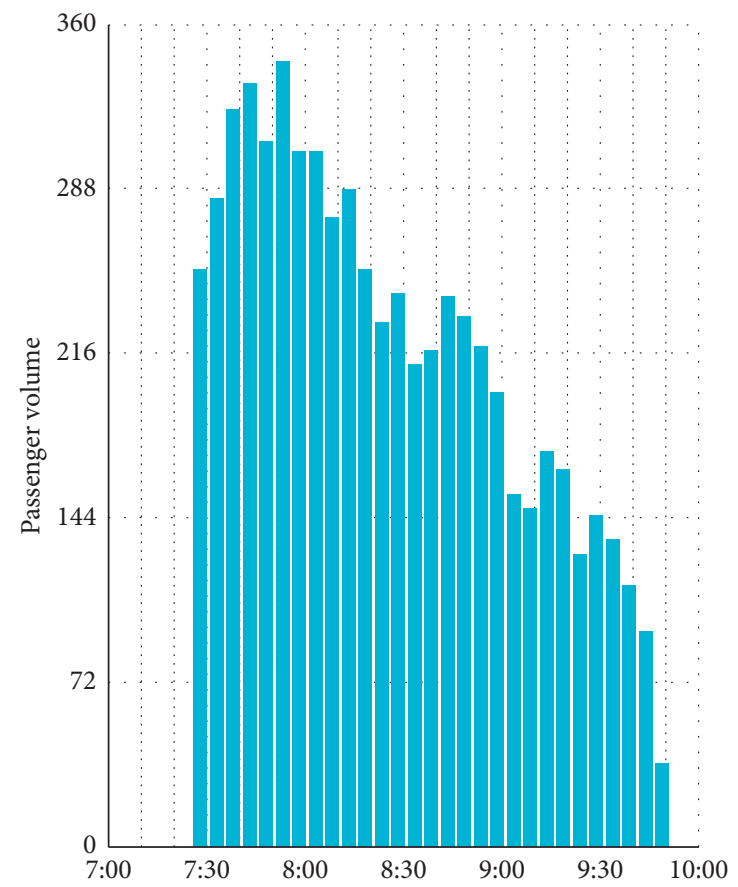

(b)

FIgURE 7: Time-varying passenger volumes at (a) TQ Station and (b) CMDX Station.

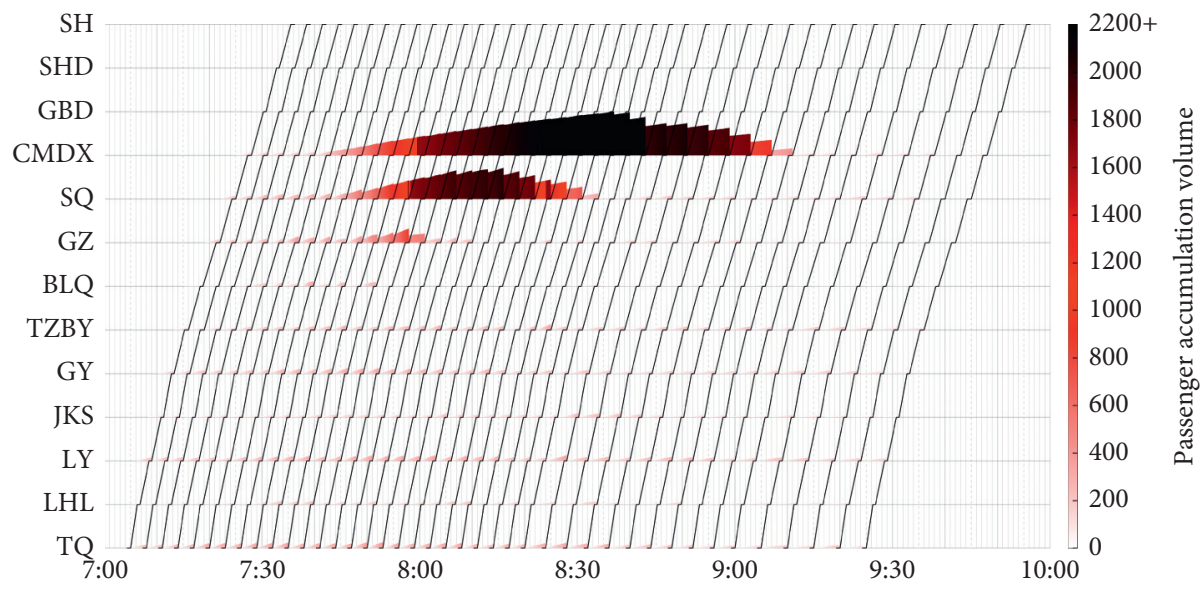

Figure 8: The timetable and passenger accumulation for NPFC. 


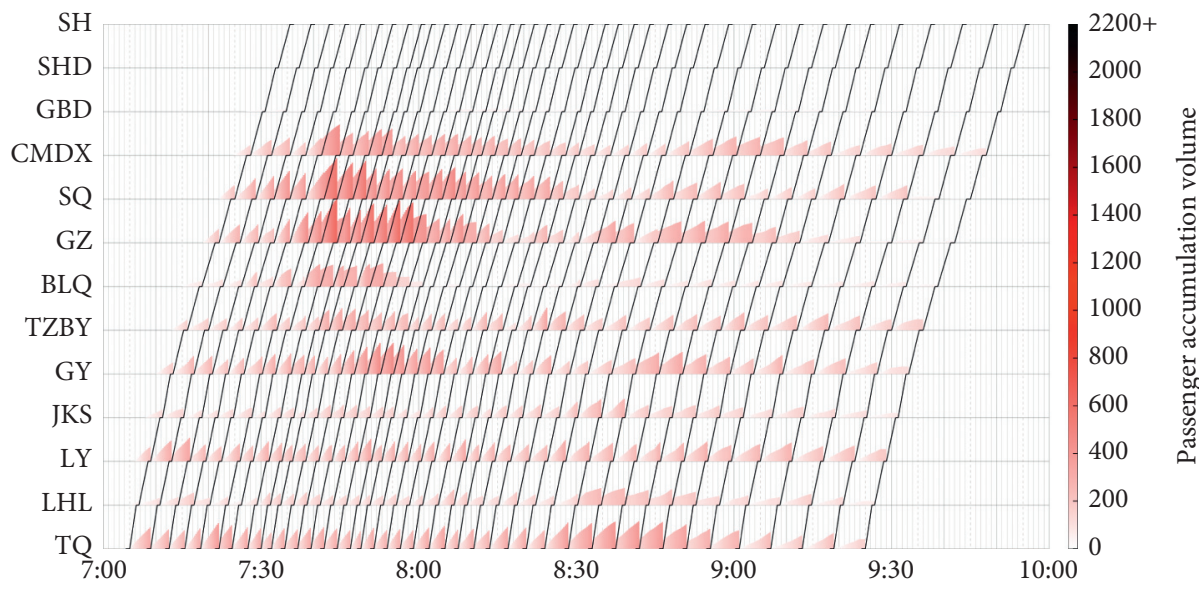

FIgURE 9: The timetable and passenger accumulation for TPFC.

capacity of several trains arriving at CMDX Station in 7: 45-8:35 has been fully occupied; therefore, passengers waiting at these two stations cannot board trains and congestion occurs, leading to serious safety and service imbalance problem.

In order to alleviate the congestion and improve the equity performance, as well as balance the train capacity utilization, the timetable and passenger flow control strategies are optimized by the TPFC model and the proposed heuristic algorithm. The optimal results show a great improvement on the imbalance indicator and train load factor equilibrium indicator with respect to 0.35 and 23.8, and the corresponding timetable and passenger accumulation situation are shown in Figure 9. Note that the optimal headways in 7:45-8:30 are smaller than the original headways, while for other time period, the headways are adjusted to be larger. This result demonstrates that the headway schedule is optimized to be more accommodative with the timedependent characteristics of passenger demands. When treating passengers at each station and the number of missed trains as one class of passengers and the cost of these passengers, respectively, we can see that costs for passengers at CMDX and SQ Stations are reduced, which results in a more equitable distribution of costs among different classes of passengers. Moreover, the passenger volume entering the platform and boarding trains at each station is well regulated by the optimal collaborative passenger flow control strategies. In detail, only control strategies at TQ and CMDX Stations are illustrated in Figure 10 . We can see that passengers are collaboratively organized to occupy the capacity of each train, and the extremely large number of trains missed by passengers at CMDX Station is prevented.

For performance comparison with and without the integrated approach, analyses on service equity, train load factor, passenger waiting time, and passenger accumulation are conducted in detail as follows.

(1) Performance Comparison on Service Equity. As shown in Figure 11, in the NPFC model, the evaluated results show that $87.1 \%$ of passengers can board the first coming train, while $8.5 \%$ of passengers have to wait at least 5 trains. Unacceptably, the maximum number of missed trains for passengers at SQ and CMDX Stations reaches 7 and 16, respectively. In comparison, the passengers missing 3 and $4+$ trains decrease by $0.8 \%$ and $8.5 \%$, while passengers missing 1 and 2 trains increase by $24.8 \%$ and $0.7 \%$ for the TPFC model. This fact demonstrates that all the passengers can get aboard within 4 missed trains, and the gap of the number of missed trains for different individuals is bridged efficiently. Although $16.2 \%$ of passengers who can board the first train previously need to wait for two trains now, we have to point out that this loss is acceptable, as passengers who need to miss at least three trains are most inequitable. Therefore, the TPFC model can shift nearly $9.3 \%$ of passengers who need to miss at least three trains to the state with lower missed trains, which improves the overall equity performance in the oversaturated Batong Line.

(2) Performance Comparison on Train Load Factor. Figure 12 shows the train load factor of each train at each segment. Intuitively, after applying the proposed TPFC model, the train loading utilization becomes more uniform and crimson segments are significantly reduced. By statistics, there are 51, 84, and 101 segments with the train load factor greater than $130 \%, 120 \%$, and $110 \%$ in Figure 10(a), while these numbers in Figure 10(b) decrease to 12, 31, and 91, respectively. We note that the segments with the train load factor greater than $100 \%$ keep the same in the TPFC model in comparison with the NPFC model. But, the optimal timetable and passenger flow control strategies can reduce the extremely overloaded segments, as well as increase train loading capacity at some segments to be more efficiently utilized, contributing to improve the transport service quality.

(3) Performance Comparison on Passenger Waiting Time. Table 7 gives the total passenger waiting time outside platforms, on platforms, and at stations. In the NPFC, we obtain the total passenger waiting time at stations $5284.5 \mathrm{~h}$, in which more than $50 \%$ is contributed by passengers waiting outside platforms. When the TPFC model is implemented, the train loading capacity and inbound passengers are collaboratively regulated. Therefore, the total 


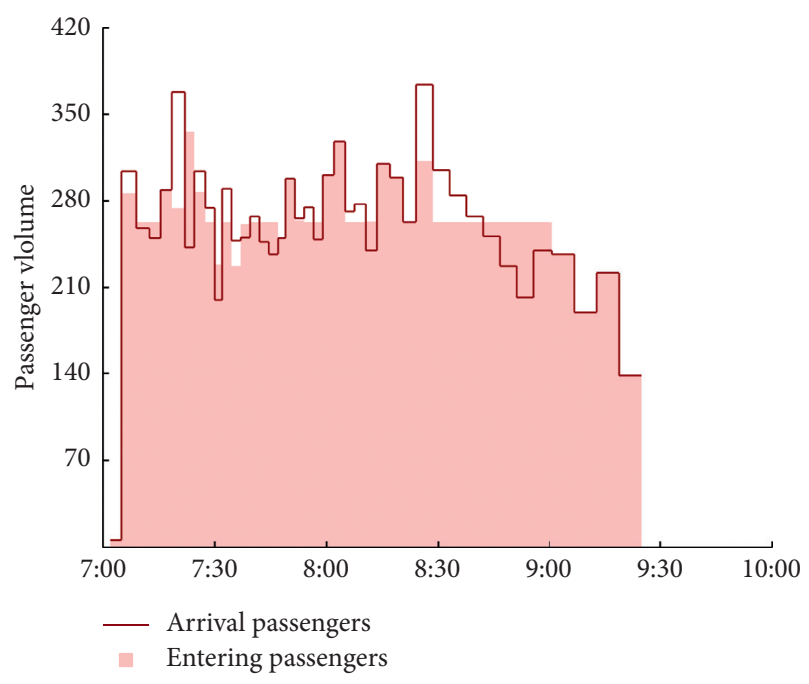

(a)

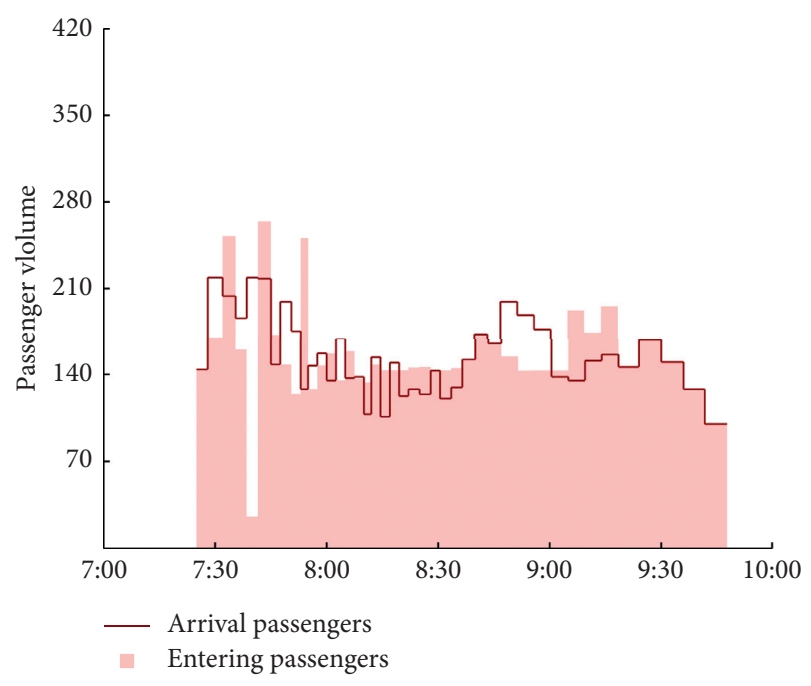

(b)

FIgure 10: Passenger flow control for (a) TQ and (b) CMDX Stations.

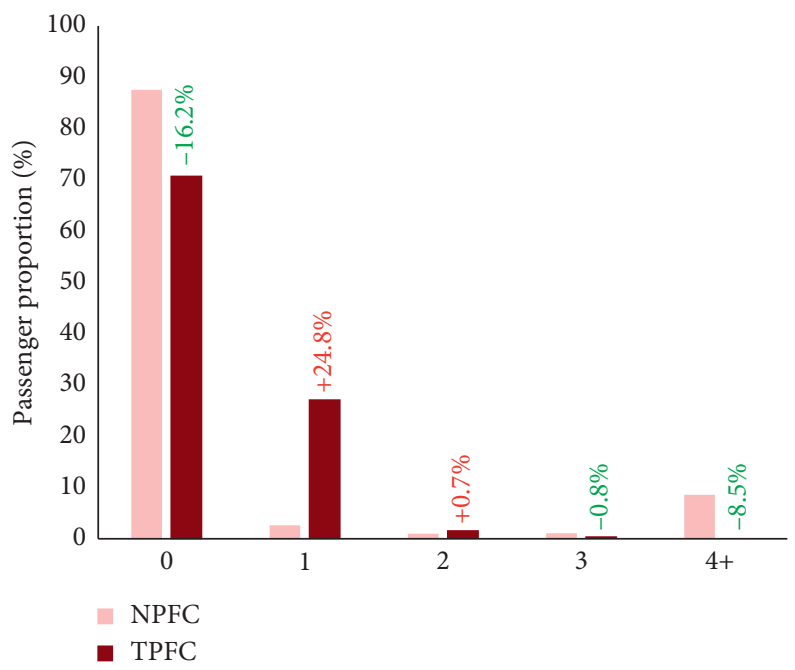

FiguRE 11: Passenger proportion of different numbers of missed trains.

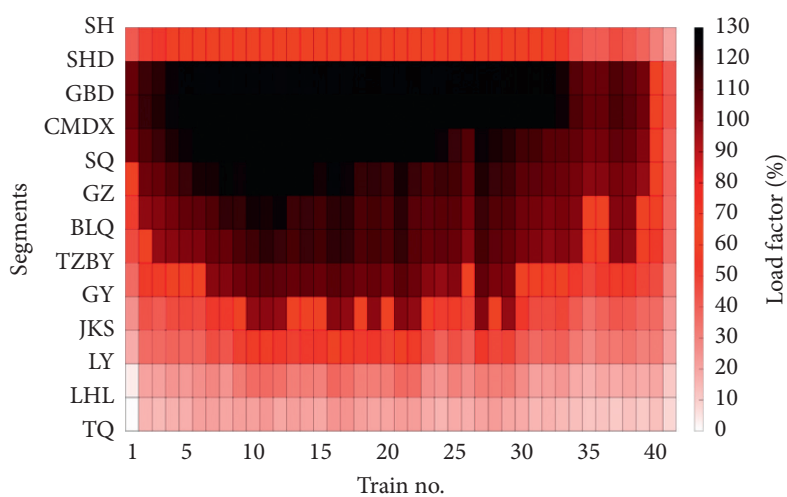

(a)

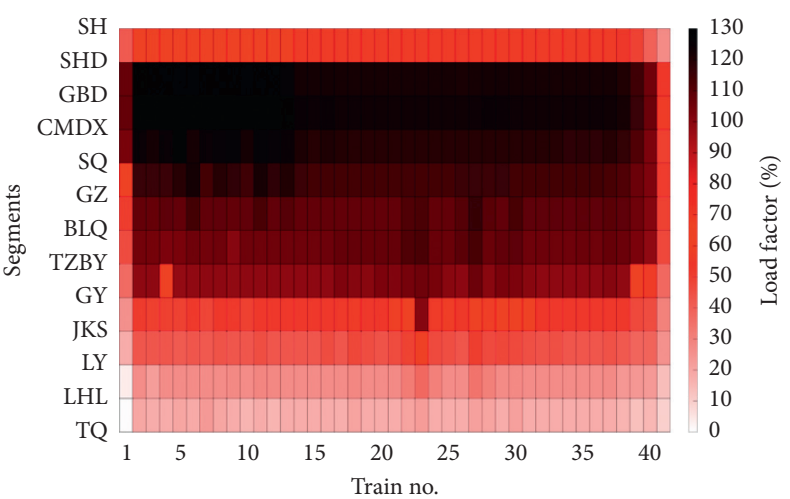

(b)

FIgURE 12: Comparison on train load factor: (a) NPFC; (b) TPFC. 
Table 7: Passenger waiting time.

\begin{tabular}{lccc}
\hline & & Waiting time (h) & \\
& Outside platforms & On platforms & At stations \\
\hline NPFC & 2850.3 & 2434.1 & 5284.5 \\
PFCT (variation \%) & $478.9(-83.20)$ & $2770.6(+13.82)$ & $3249.5(-38.51)$ \\
\hline
\end{tabular}

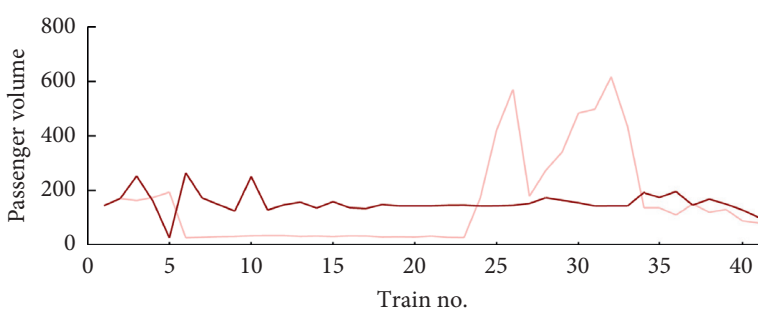

- TPFC

NPFC

(a)

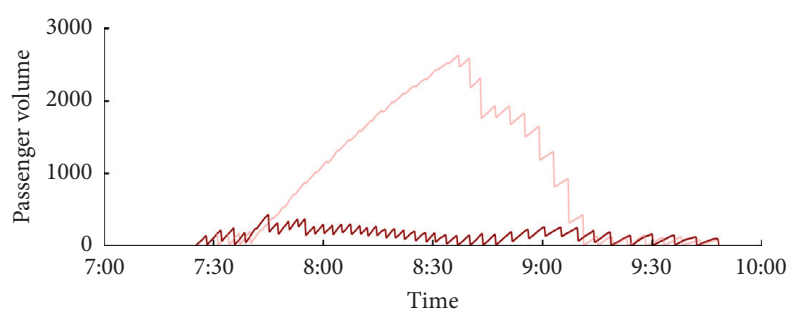

- TPFC

NPFC

(b)

Figure 13: Passenger loading dynamics at CMDX Station: (a) boarding passengers; (b) accumulated passengers.

passenger waiting time at stations decreases by $38.51 \%$, as the passengers stranded outside platforms are reduced significantly. Likewise, the passenger waiting time on platform increases slightly because more passengers are allowed to enter platforms to wait for trains with less queuing time at waiting areas.

(4) Performance Comparison on Passenger Accumulation. Figure 13 demonstrates the detailed comparison of boarding passengers for each train and accumulated passengers at CMDX Station with the NPFC and TPFC models. For NPFC, only few passengers can get trains before 8:30 because the involved trains are fully occupied by passengers boarding at upstream stations. This leads to a fast accumulation at CMDX Station, and the maximum accumulation number is approximately 2633 at 8:37. Later, the passenger demands at upstream stations decrease to below the train capacity, then the stranded passengers are transported, and congestion fades away gradually. As for TPFC, the loading capacity of each train is partly reserved for passengers at CMDX Station according to the optimized strategies, so the number of passengers boarding each train is relatively balanced and the high accumulation is prevented. Under this circumstance, the maximum number of passenger accumulation at CMDX Station is 437 at $7: 45$, which is insufficient to result in a congestion situation.

\section{Conclusions}

On an oversaturated urban rail line, some passengers at downstream stations may wait for more trains than passengers at upstream stations, leading to a service imbalance problem. To improve the overall service quality, this paper studied the equity-oriented train timetabling as well as collaborative passenger flow control. A multiobjective mixed-integer linear programming model is formulated, and the proposed model aimed to minimize an imbalance indicator related to the number of missed trains, as well as the train load factor equilibrium indicator. The linear weighting method was used to convert the multiobjective model into a single-objective model, which was later decomposed into two subproblems. Based on the decomposition, an iterative heuristic algorithm integrating the tabu search and Gurobi solver was proposed to solve the model.

A simple case and a real-world case of on Beijing Subway Batong Line were conducted to demonstrate the effectiveness and efficiency of the proposed integrated model and heuristic algorithm. In the experimental results, it was verified that optimized heterogeneous headways and passenger flow control strategies can balance the train loading capacity utilization for each train and reduce the number of passengers suffering the maximum number of missed trains. Since the train loading capacities were redistributed according to spatiotemporal demands with optimal train and passenger regulations, high accumulation situations were prevented and total passenger waiting time at stations was reduced significantly.

This paper preliminarily explored the integrated optimization of the train timetabling and passenger flow control for one direction of an oversaturated urban rail line, while urban rail lines are usually bidirectional. In this case, the proposed approach might not be the optimal one. One of our future researches could be to study a bidirectional urban rail line and implement a more applicable equity-oriented timetable considering train connections. In addition, only heterogeneous headways are employed in the train scheduling process in this study. Other train operation approaches, such as skip-stop patterns and shortturning routes, are likely lead to more safe and efficient operations of urban rail networks, which is another 
significant topic for future researches. Finally, social equity is extended and narrowed down to the service imbalance problem, and discussions on distribution of benefits or costs among different class of passengers are insufficient. It is worthy in the further research to classify passengers and qualify benefits/costs more clearly and explore their connections on equity.

\section{Data Availability}

The data used to support the findings of this study are included within the article.

\section{Conflicts of Interest}

The authors declare that there are no conflicts of interest regarding the publication of this paper.

\section{Acknowledgments}

This research was funded by the National Natural Science Foundation of China (71971021 and 71621001) and the Fundamental Research Funds for the Central Universities (2019JBM334).

\section{References}

[1] China Association of Metros, Report on the Development of Urban Rail Transit Operation in China (2018-2019), Social Sciences Academic Press (China), Beijing, China, 2019.

[2] T. Litman, "Evaluating transportation equity," Word Transport Policy and Practice, vol. 8, no. 2, pp. 50-65, 2002.

[3] R. Camporeale, L. Caggiani, and M. Ottomanelli, "Modeling horizontal and vertical equity in the public transport design problem: a case study," Transportation Research Part A: Policy and Practice, vol. 125, pp. 184-206, 2019.

[4] K. Lucas, K. Martens, F. D. Ciommo et al., Measuring Transport Equity, Elsevier Science Publishing, Amsterdam, Netherlands, 2019.

[5] A. El-Geneidy, D. Levinson, E. Diab, G. Boisjoly, D. Verbich, and C. Loong, "The cost of equity: assessing transit accessibility and social disparity using total travel cost," Transportation Research Part A: Policy and Practice, vol. 91, pp. 302-316, 2016.

[6] L. Caggiani, R. Camporeale, and M. Ottomanelli, "Facing equity in transportation network design problem: a flexible constraints based modelflexible constraints based model," Transport Policy, vol. 55, pp. 9-17, 2017.

[7] A. Karner, "Assessing public transit service equity using route-level accessibility measures and public data," Journal of Transport Geography, vol. 67, pp. 24-32, 2018.

[8] K. Kelobonye, G. McCarney, J. Xia, M. S. H. Swapan, F. Mao, and H. Zhou, "Relative accessibility analysis for key land uses: a spatial equity perspective," Journal of Transport Geography, vol. 75, pp. 82-93, 2019.

[9] X. Luan, F. Corman, and L. Meng, "Non-discriminatory train dispatching in a rail transport market with multiple competing and collaborative train operating companies," Transportation Research Part C: Emerging Technologies, vol. 80, pp. 148-174, 2017.

[10] M. Zhong, "Models and solution algorithms for equitable resource allocation in air traffc flow management," $\mathrm{Ph}$.
D. Thesis, University of Maryland, College Park, MD, USA, 2012.

[11] E. Barrena, D. Canca, L. C. Coelho, and G. Laporte, "Exact formulations and algorithm for the train timetabling problem with dynamic demand," Computers \& Operations Research, vol. 44, pp. 66-74, 2014.

[12] E. Barrena, D. Canca, L. C. Coelho, and G. Laporte, "Singleline rail rapid transit timetabling under dynamic passenger demand," Transportation Research Part B: Methodological, vol. 70, pp. 134-150, 2014.

[13] L. Sun, J. G. Jin, D.-H. Lee, K. W. Axhausen, and A. Erath, "Demand-driven timetable design for metro services," Transportation Research Part C: Emerging Technologies, vol. 46, pp. 284-299, 2014.

[14] H. Niu and X. Zhou, "Optimizing urban rail timetable under time-dependent demand and oversaturated conditions," Transportation Research Part C: Emerging Technologies, vol. 36, pp. 212-230, 2013.

[15] Y. Gao, L. Kroon, M. Schmidt, and L. Yang, "Rescheduling a metro line in an over-crowded situation after disruptions," Transportation Research Part B: Methodological, vol. 93, pp. 425-449, 2016.

[16] P. Shang, R. Li, Z. Liu, L. Yang, and Y. Wang, "Equity-oriented skip-stopping schedule optimization in an oversaturated urban rail transit network," Transportation Research Part C: Emerging Technologies, vol. 89, pp. 321-343, 2018.

[17] Z. Li, B. Mao, Y. Bai, and Y. Chen, "Integrated optimization of train stop planning and scheduling on metro lines with express/local mode," IEEE Access, vol. 7, pp. 88534-88546, 2019.

[18] S. Li, R. Xu, and K. Han, "Demand-oriented train services optimization for a congested urban rail line: integrating short turning and heterogeneous headways," Transportmetrica A: Transport Science, vol. 15, no. 2, pp. 1459-1486, 2019.

[19] Y. H. Zhou, Y. Bai, J. J. Li et al., "Integrated optimization on train control and timetable to minimize net energy consumption of metro lines," Journal of Advanced Transportation, vol. 2018, Article ID 7905820, 19 pages, 2018.

[20] M. Gallo, B. Montella, and L. D'Acierno, "The transit network design problem with elastic demand and internalisation of external costs: an application to rail frequency optimisation," Transportation Research Part C: Emerging Technologies, vol. 19, no. 6, pp. 1276-1305, 2011.

[21] The Beijing News, Ninety-Six Stations of Beijing Subway Implement Passenger Flow Control Strategies in Their Daily Operations, http://www.bjnews.com.cn/news/2018/01/07/ 471465.html.

[22] Z. Jiang, W. Fan, W. Liu, B. Zhu, and J. Gu, "Reinforcement learning approach for coordinated passenger inflow control of urban rail transit in peak hours," Transportation Research Part C: Emerging Technologies, vol. 88, pp. 1-16, 2018.

[23] Z. Zhang, L. Jia, Y. Qin, and T. Yun, “Optimization-based feedback control of passenger flow in subway stations for improving level of service," Transportation Letters, vol. 11, no. 8, pp. 413-424, 2019.

[24] X.-y. Xu, J. Liu, H.-y. Li, and M. Jiang, "Capacity-oriented passenger flow control under uncertain demand: algorithm development and real-world case study," Transportation Research Part E: Logistics and Transportation Review, vol. 87, pp. 130-148, 2016.

[25] S. K. Chen, S. Liu, X. Xiao et al., "M/G/c/c-based model of passenger evacuation capacity of stairs and corridors in metro stations," Journal of the China Railway Society, vol. 34, no. 1, pp. 7-12, 2012. 
[26] L. L. Wang, X. D. Yan, and Y. Wang, "Modeling and optimization of collaborative passenger control in urban rail stations under mass passenger flow," Mathematical Problems in Engineering, vol. 2015, Article ID 786120, 8 pages, 2015.

[27] J. Yang, J. G. Jin, J. Wu, and X. Jiang, "Optimizing passenger flow control and bus-bridging service for commuting metro lines," Computer-Aided Civil and Infrastructure Engineering, vol. 32, no. 6, pp. 458-473, 2017.

[28] Q. R. Zou, X. M. Yao, P. Zhao et al., "Managing recurrent congestion of subway network in peak hours with station inflow control," Journal of Advanced Transportation, vol. 2018, Article ID 6931025, 16 pages, 2018.

[29] X. Xu, H. Li, J. Liu, B. Ran, and L. Qin, "Passenger flow control with multi-station coordination in subway networks: algorithm development and real-world case study," Transportmetrica B: Transport Dynamics, vol. 7, no. 1, pp. 446-472, 2019.

[30] J. Shi, L. Yang, J. Yang, F. Zhou, and Z. Gao, "Cooperative passenger flow control in an oversaturated metro network with operational risk thresholds," Transportation Research Part C: Emerging Technologies, vol. 107, pp. 301-336, 2019.

[31] J. Shi, L. Yang, J. Yang, and Z. Gao, "Service-oriented train timetabling with collaborative passenger flow control on an oversaturated metro line: an integer linear optimization approach," Transportation Research Part B: Methodological, vol. 110, pp. 26-59, 2018.

[32] Z. Jiang, J. Gu, W. Fan, W. Liu, and B. Zhu, "Q-learning approach to coordinated optimization of passenger inflow control with train skip-stopping on a urban rail transit line," Computers \& Industrial Engineering, vol. 127, pp. 1131-1142, 2019.

[33] R. M. Liu, S. K. Li, and L. X. Yang, "Collaborative optimization for metro train scheduling and train connections combined with passenger flow control strategy," Omega, vol. 90, Article ID 101990, 2020.

[34] S. Li, M. M. Dessouky, L. Yang, and Z. Gao, "Joint optimal train regulation and passenger flow control strategy for highfrequency metro lines," Transportation Research Part B: Methodological, vol. 99, pp. 113-137, 2017.

[35] J. J. Shi, "Calculation and optimization for the conveying capacity utilization of urban rail transit," $\mathrm{Ph}$. D. thesis, Beijing Jiaotong University, Beijing, China, 2017. 